\title{
El Estudio de Propiedades Geométricas de Poliedros Regulares: Una Propuesta Mediada con Tecnología Digital
}

\author{
The study of geometric properties of regular polyhedra: a digitally \\ mediated approach

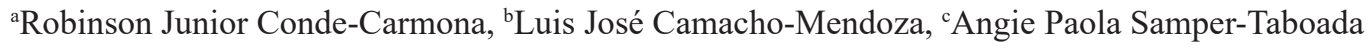 \\ ${ }^{a} \mathrm{PhD}$ (C) en Educación Matemática, rjconde@mail.uniatlantico.edu.co, https://orcid.org/0000-0002-7421-1754, Universidad del \\ Atlántico, Barranquilla, Colombia
bMaestrante en Ciencias Físicas, ljcamacho@mail.uniatlantico.edu.co, https://orcid.org/0000-0002-5865-93137, colegio I.E.D. Nuestra señora de las nieves, Barranquilla, Colombia
`Maestrante en Educación Matemática, apsamper@mail.uniatlantico.edu.co, https://orcid.org/0000-0003-1446-3308, Liceo Cervantes, \\ Barranquilla, Colombia
}

Forma de citar: Conde Carmona, R. J., Camacho Mendoza, L. J., \& Samper Taboada, A. P. . (2021). El estudio de propiedades geométricas de poliedros regulares: una propuesta mediada con tecnología digital.

Eco Matemático, 12 (2), 71-86

Recibido: $16 / 04 / 2021$

Aceptado: 9/05/2021

\section{Palabras clave}

Tecnología digital; situaciones a-didácticas; etapas de la geometría escolar; ingeniería didáctica; poliedros regulares

\begin{abstract}
Resumen: La investigación tuvo como objetivo el diseño e implementación de situaciones a-didácticas haciendo uso de las etapas de la geometría escolar y software de geometría dinámica como medio didáctico; para el aprendizaje de las propiedades de poliedros regulares. Las situaciones diseñadas fueron aplicadas a estudiantes de quinto grado de escolaridad en básica primaria. La propuesta estuvo fundamentada en la teoría de las Situaciones Didácticas; y en el proceso metodológico de investigación y análisis fue referente la Ingeniería Didáctica. Los resultados de la investigación permitieron determinar aspectos importantes en el desarrollo de las etapas de la Geometría escolar y las competencias de comunicación, razonamiento y solución de problemas geométricos; además de ratificarse el potencial didáctico que las Tecnologías digitales ofrecen en los procesos de enseñanza y aprendizaje de la Geometría
\end{abstract}

*Autor para correspondencia: rjconde@mail.uniatlantico.edu.co

Doi: https://doi.org/10.22463/17948231.3234 


\section{Keywords}

Digital technology; a didactic situations; stages of school geometry; didactic Engineering; regular polyhedra

\begin{abstract}
The objective of the research was the design and implementation of a-didactic situations making use of the stages of school geometry and dynamic geometry software as a didactic means; for learning the properties of regular polyhedra. The designed situations were applied to fifth grade students in elementary school. The proposal was based on the theory of Didactic Situations; and in the methodological process of research and analysis, Didactic Engineering was a reference. The results of the investigation allowed to determine important aspects in the development of the stages of school geometry and the competences of communication, reasoning and solution of geometric problems; in addition to ratifying the didactic potential that digital technologies offer in the teaching and learning processes of Geometry.
\end{abstract}

\section{Introducción}

Las Tecnologías de la Información y la Comunicación (TIC) para la enseñanza y aprendizaje de la geometría es un tema de investigación fundamental en el medio de acción de la educación matemática de las últimas décadas. Las nuevas tecnologías, tales como simulaciones, entornos de aprendizaje en línea, y los softwares están siendo más utilizados en la práctica educativa, favoreciendo el desarrollo continuo del conocimiento (Mulder et al. 2012; Van Dijk y Lazonder 2013; Rodríguez, López, y Mortera, 2017). En este sentido, el uso de los medios digitales puede mejorar la calidad de la enseñanza, los resultados del aprendizaje, el interés, la creatividad, el trabajo colaborativo y las estrategias de aprendizaje de los alumnos en una nueva era digital (Badia, Chumpitaz, Vargas, y Suárez, 2016).

Aun cuando existen una gama de proyectos de investigación que presentan experiencias para demostrar las bondades del uso de las nuevas tecnologías en educación, también es importante hacer notar que en otro tanto de estos trabajos publicados no se evidencia una orientación didáctica y teórica que posibilite analizar los procesos de aprendizaje presentes en los actos pedagógicos que hacen uso de tecnología, y qué aspectos de dichas experiencias son rescatables como exitosos (Acosta, Monroy y Rueda, 2010; Conde-Carmona, Samper-Taboada, Camacho-Mendoza, 2020). Por tal razón, las investigaciones en educación con ayuda de la tecnología como medio didáctico deben fundamentarse en teorías específicas, en especial en el campo de la educación matemática.

En el caso particular de la enseñanza de la geometría, el uso de tecnología digital aún no se posiciona de forma masiva dentro del desarrollo de los procesos de enseñanza. Esta situación se relaciona con que los docentes carecen de habilidades TIC para hacer frente a los desafíos de la educación del presente siglo; en algunas investigaciones se revela que los maestros ven difícil la utilización de las nuevas tecnologías en el aula (Janssen y Lazonder, 2015, Conde-Carmona y Fontalvo, 2019). Otra actitud que explica esta situación es la falta de competencias para adaptar sus estilos de enseñanza con las nuevas tecnologías digitales, los maestros pueden sentirse amenazados por el cambio de una metodología tradicional en la cual están conformes y por tanto se resisten a ello, así lo describen Lucumi y Gonzales (2015).

Según los expuesto anteriormente, los docentes deben apropiarse de las herramientas digitales que le ofrece el presente siglo, que ayuden a potenciar las competencias de los estudiantes, con el fin de idear estrategias de solución a problemas ya establecidos en los procesos propios de enseñar y aprender y por consiguiente, también se logre mejorar la actitud, motivación, interés, disfrute y entusiasmo de los estudiantes. En otras palabras, el docente de matemáticas requiere un cambio en su metodología 
de enseñanza, que investigue su propia práctica educativa (Padilla, Conde-Carmona, 2021) en aras a optimizar la misma, lo que exige una renovación en el conocimiento profesional del docente.

Por su parte, los avances tecnológicos del presente siglo están aportando entornos de Geometría Dinámica que permiten procesos de visualización, construcción, justificación y demostración geométrica, habilidades necesarias para: el desarrollo de la geometría de las formas, las construcciones, justificaciones y demostraciones, conocidas estas 4 como las etapas de la Geometría escolar, las cuales son definidas y usadas por autores como Acosta, (2017) para contribuir el desarrollo del pensamiento espacial en los estudiantes. Además, en unos de sus trabajos concluye que fracasos de la geometría escolar en la actualidad son debidos al desarrollo de forma inadecuada de las etapas de la geometría en los niveles escolares, específicamente en la básica primaria.

Lo descrito anteriormente, abre las puertas al estudio de la mediación de las tecnologías digitales, en especial la implantación de software de Geometría Dinámica en el proceso de desarrollo de competencias propias del saber matemático que enmarcan el estudio de las etapas de la geometría escolar en la básica primaria, tomando como objeto de estudio investigativo a dos instituciones públicas en la ciudad de Barranquilla-Colombia, en el desarrollo competencias a dentro del estudio propiedades de poliedros regulares en estudiantes de quinto grado. Estas competencias según el documento de las Matrices de Referencia el Ministerio de Educación Nacional Colombiano son la comunicación, razonamiento y resolución de Problemas (MEN, 2015).

Por tanto, el desarrollo de competencias requiere la creación de un proceso de aprendizaje activo/constructivo, auto regulado, situado y colaborativo, donde se considere que es el estudiante quien construye significados y conocimientos matemáticos; se necesita poner atención en el rol del profesor en el aula, el tipo de tareas a proponer y el ambiente de aprendizaje a generar (Vargas, Escalante y Carmona, 2018). Es por esta razón que el desarrollo de competencias geométricas durante las etapas de la geometría escolar no es un acto independiente, apartada de la naturaleza de cada una, sino un proceso que integra el desarrollo del pensamiento espacial. Además, el docente debe generar el entorno de aprendizaje y las situaciones problemas adecuadas para lograr en los estudiantes un aprendizaje por adaptación o situación a-didáctica.

Por consiguiente, la hipótesis del presente trabajo es que el desarrollo de competencias matemáticas, en especial, de competencias geométricas se potenciarían en los estudiantes de quinto grado a través del diseño e implementación de situaciones a-didácticas mediadas por tecnologías digitales. Para constatar tal hipótesis se diseñaron situaciones a-didácticas teniendo en cuenta las de la Ingeniería Didáctica como diseño de investigación. Este diseño contó con un análisis preliminar, análisis a priori de las actividades, experimentación y análisis a posteriori, los cuales serán explicados en posteriores secciones del presente documento.

En tal sentido, para hablar de competencias se inicia con la competencia de comunicación, la cual involucra los procesos de visualización de propiedades geométricas, y se dan durante la etapa de la geometría de las formas y construcciones. Por consiguiente, la visualización es un proceso de desconfiguración o reconfiguración cuyo propósito es detectar, percibir o evocar propiedades geométricas presentes en una representación gráfica. Esto obliga, a que el estudio de la visualización de propiedades geométricas debe ser parte fundamental en los currículos escolares, más específicamente en la educación básica primaria, visto como la introducción de las bases para un desarrollo satisfactorio del pensamiento espacial. 
Cabe resaltar, que el potencial de la Geometría Dinámica permite un equilibrio de los procesos de visualización y los procesos de justificación para un aprendizaje significativo de la geometría (Camargo y Acosta, 2012). Estos procesos de justificación involucran el desarrollo de la competencia de razonamiento y hacen parte de la segunda etapa de la geometría escolar o geometría de las justificaciones. En cuanto a la competencia de solución de problemas, es la más compleja pues involucra la apropiación de las competencias de comunicación y razonamiento; esta competencia se desarrolla durante la etapa de la geometría de las demostraciones. Cada una de las etapas de la geometría escolar se especifican en el siguiente apartado.

Por lo anteriormente expuesto,

\section{Referentes Teóricos}

\section{Etapas de la geometría escolar}

El estudio de las etapas de la geometría escolar se fundamenta en los trabajos de Acosta (2017). La primera etapa es la geometría de las formas, en la cual se debe desarrollar la habilidad la visualización geométrica. La visualización integra los procesos por medio de los cuales se obtienen conclusiones, a partir de las representaciones de los objetos bi o tridimensionales y de las relaciones o transformaciones observadas en construcciones y manipulaciones (Clements y Battista, 1992).

La segunda etapa es la geometría de las construcciones, en la cual se conservan aspectos generales de primera etapa y enmarca un tránsito hacia la tercera etapa de las justificaciones. Para Acosta (2017) en la etapa de la geometría de las construcciones las figuras se consideran como formas compuestas por objetos básicos (vértices y segmentos), con relaciones espaciales entre ellos como el paralelismo, perpendicularidad, equidistancia, entre otras. En esta etapa los estudiantes deben identificar las propiedades de una figura como elementos fundamentales.

La geometría de las justificaciones es la tercera etapa de la Geometría escolar y se da inmediatamente el estudiante es capaz de comenzar a dar explicaciones propias acerca de una construcción realizada a partir de sus propiedades geométricas. Aunque el cambio parezca un poco sutil, el proceso se da por la necesidad de justificar los hechos geométricos en una construcción. Los estudiantes necesitan desarrollar habilidades que les permitan construir significados con base a representaciones de formas, gráficos, visualizaciones y simulaciones, y lo más importante, que desarrollen la capacidad de crítica de dar argumentos (Squire y Jan, 2007).

\section{Teoría de las situaciones didácticas en la enseñanza de la geometría}

La presente investigación se desarrolló bajo el enfoque de la Teoría de las Situaciones Didácticas (TSD) detallada por Acosta (2017) quien cita a Brousseau (1986). A continuación, se describen los conceptos que servirán para orientar la teoría utilizada: Aprendizaje por adaptación, Situación a-didáctica y Situación didáctica.

\section{Aprendizaje por adaptación}

El concepto central de la TSD es el de aprendizaje por adaptación. Se entiende el aprendizaje por adaptación como aquel aprendizaje que se produce sin la mediación de un profesor como producto de la interacción entre un sujeto y un medio. Para el caso particular de la investigación, estudiantes cumplieron el papel de los objetos de estudio y el software de Geometría Dinámica GeoGebra es el medio. En el aprendizaje por adaptación el sujeto tiene una intención, una necesidad o un objetivo, y para alcanzarlo realiza una acción sobre el medio. Este medio reacciona a esa acción; esta reacción se conoce como retroacción. El sujeto interpreta esta 
retroacción para poder decidir si alcanzó o no el propósito.

\section{Situación a-didáctica y situación didáctica}

El aprendizaje por adaptación no contempla la intervención de un profesor; sin embargo, en la teoría de la TSD el rol del profesor es muy importante, puesto que es el encargado de crear la intención en el estudiante y preparar correctamente el medio. El profesor anticipar las posibles acciones del estudiante y las retroacciones del medio para garantizar que puedan ser interpretadas por el estudiante, con el fin de validar o invalidar sus acciones, y que de esta manera se dé un aprendizaje por adaptación. Por lo anterior, es el docente el encargado de generar las condiciones necesarias y plantear los problemas adecuados para lograr en los estudiantes un aprendizaje por adaptación; a este proceso se lo conoce como situación a-didáctica.
Para Acosta (2010) una situación a-didáctica solo puede comprenderse en relación con una situación didáctica que es una situación normal de clase. Una situación es didáctica cuando el profesor tiene la intención de enseñar al estudiante un saber matemático dado. Una situación es a-didáctica cuando se da interacción entre un sujeto y un medio para resolver un problema. Como el medio es impersonal, no tiene ninguna intención didáctica: no desea enseñarle nada al alumno. Por eso este tipo de situación recibe el nombre de a-didáctica. Aunque, podría pensarse que estas dos situaciones están totalmente en oposición, puesto que una necesita del profesor y la otra no, según la TSD se da una interacción de estas dos situaciones, en la que la situación a-didáctica puede ser parte de una situación didáctica. En la Figura 1 se ilustra esta interacción.

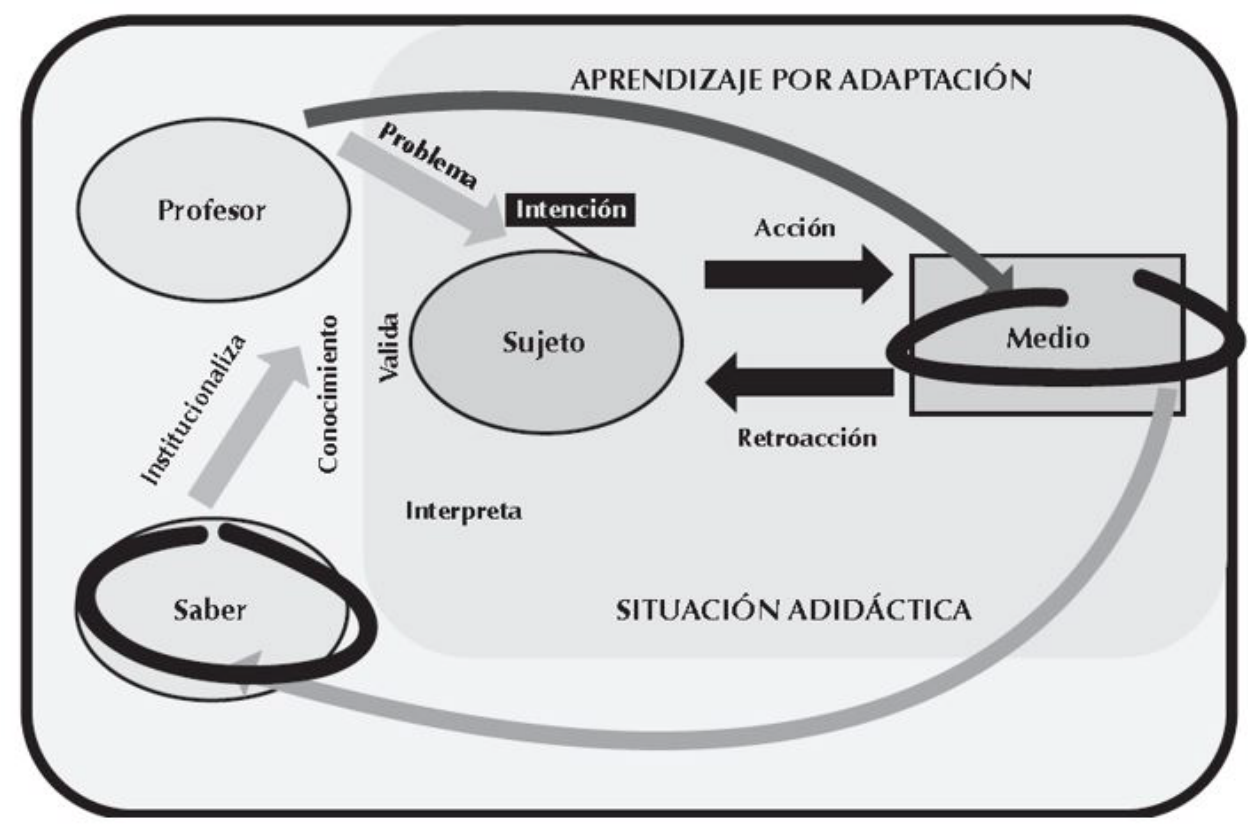

Figura 1. Teoría de las situaciones Didácticas. Tomado de Acosta (2017).

Se visualiza entonces, que al interior de la situación didáctica hay una situación a-didáctica que el profesor utiliza para que los alumnos construyan un conocimiento, al cual podrá referirse para exponer el saber. La función principal del profesor es la de preparar la situación a-didáctica: seleccionar cuidadosamente el medio y el problema que planteará a los alumnos. Mientras se lleva a cabo la situación a-didáctica, 
el profesor se abstiene de comunicar el saber a los alumnos, pues de esa manera impediría que se realice un aprendizaje por adaptación. Esto no quiere decir que el profesor no deba intervenir durante la situación a-didáctica, sino que su intervención debe limitarse a animar al alumno a resolver el problema, hacerle tomar conciencia de las acciones que puede realizar y de las retroacciones del medio, pidiéndole que sea él mismo quien decida si resolvió o no el problema (validación). Este proceso recibe el nombre de devolución. Una vez terminada la situación a-didáctica, el profesor retoma su responsabilidad de enseñar, explicitando las relaciones entre el conocimiento construido en la situación a-didáctica y el saber que desea comunicar (fase de institucionalización).

\section{Metodología}

Se optó como diseño de investigación, la Ingeniería Didáctica (ID) propuesta por Artigue (1995); utilizada por Acosta (2017) y Rueda, Niño y Acosta (2013) como marco metodológico de investigaciones que incorporan el uso de tecnologías digitales para su desarrollo y análisis; en particular, en el presente trabajo se utilizó la Ingeniería Didáctica para el análisis de una secuencia de actividades mediadas por software de Geometría Dinámica.

La metodología de investigación está fundamentada por dos etapas A y B. La Etapa A corresponde a la etapa de la selección de la muestra objeto de estudio a través de la aplicación de una prueba diagnóstico. Los datos recolectados se convierten en información necesaria para el objeto de estudio de la investigación (Hernández, Fernández y Baptista, 2014). La Etapa B responde a la implementación y análisis de una secuencia de actividades mediante las fases metodológicas de la ID.

De acuerdo con todo lo anterior, para que una situación a-didáctica pueda ser utilizada para la enseñanza de un saber determinado, el conocimiento producido por la interacción con el medio debe tener una relación con el saber a enseñar, en tal sentido la presente investigación tomó como marco de referencia la TSD para desarrollar competencias en estudiantes de quinto grado de escolaridad, se incorporan las tecnologías digitales usando el software de Geometría Dinámica GeoGebra como medio didáctico en el desarrollo de las etapas de la geometría escolar en el estudio de propiedades de poliedros regulares.

Etapa A, Selección de la muestra. Se realizó la prueba inicial a los estudiantes grado $5^{\circ}$ de dos instituciones de carácter público de la ciudad de barranquilla-Colombia, con edades de 10 a 11 años, con el objetivo de conocer el nivel de desarrollo de competencias en las etapas de la geometría escolar en el estudio de poliedros regulares. La aplicación de la prueba sirvió como instrumento para la recolección de la información primaria en el proceso investigativo. La muestra objeto de estudio es homogénea y se estableció por conveniencia, obedeciendo a criterios e intereses de la investigación y su utilización práctica para fines del estudio. El análisis de la prueba inicial, determinó que el estudio se llevaría a cabo con 30 estudiantes entre ambos Centros Educativos. Los estudiantes seleccionados presentaron bajos niveles en el desarrollo de la prueba inicial; la cual tuvo como objetivo identificar las dificultades que presentan los estudiantes en el desarrollo de las competencias de comunicación, razonamiento y solución de problemas en el estudio de propiedades de poliedros regulares.

Etapa B, Ingeniería Didáctica. Se establecieron las siguientes fases metodológicas de la Ingeniería Didáctica propuesta por Artigue (1995):

Fase1. Análisis preliminar: presentación de saberes matemáticos necesarios para el desarrollo de la investigación, los cuales son elementos básicos como paralelismo, perpendicularidad, polígono regular y convexidad. Como saber propio a trabajar 
se desarrolló el desarrollo de competencias desde el estudio de poliedros regulares y sus propiedades.

Fase 2. Análisis a priori de las actividades: Se realizó un análisis de las posibles respuestas, aptitudes y estrategias que utilizará el estudiante al momento de desarrollar cada una las actividades que se le presenten con respecto a los poliedros regulares y sus propiedades, buscando siempre el desarrollo de competencias geométricas durante las etapas de la Geometría escolar.

Fase 3. Experimentación: Se implementó la propuesta didáctica mediada por la Geometría Dinámica en el desarrollo de competencias durante las etapas de la geometría escolar.
Fase 4. Análisis a posteriori de las actividades y evaluación de la propuesta: en esta última fase de la Ingeniería Didáctica se realizó un análisis sobre los datos recogidos de la experimentación (fase anterior) y confrontación final de los resultados de la investigación.

Durante la intervención didáctica realizó la de recolección de la información a través de una prueba escrita aplicada a los estudiantes y la transcripción de videos y/o audios de las actividades. Las convenciones utilizadas para las transcripciones se muestran en la tabla I.

Tabla I. Convenciones de las transcripciones

\begin{tabular}{|c|c|}
\hline Convención & Descripción \\
\hline $\mathbf{E}$ & $\begin{array}{l}\text { Representa un estudiante cualquiera de la muestra, si son varios } \\
\text { estudiantes en una misma intervención se coloca el subíndice } 1,2,3,3 \text {, } \\
\text { entre otros, según el caso. }\end{array}$ \\
\hline $\mathbf{G}$ & $\begin{array}{l}\text { Representa grupos de estudiantes, debido a que algunas actividades se } \\
\text { desarrollaron en parejas o grupos de } 3 \text { estudiantes. }\end{array}$ \\
\hline $\mathbf{D}$ & $\begin{array}{l}\text { Representa al docente investigador, quien es el mediador durante todas las } \\
\text { actividades realizadas. }\end{array}$ \\
\hline $\begin{array}{l}\text { Letra en } \\
\text { negrita azul }\end{array}$ & $\begin{array}{l}\text { Describir lo que decían o justificaban los estudiantes de forma oral, o } \\
\text { también señalando la pantalla del computador. }\end{array}$ \\
\hline $\begin{array}{l}\text { Letra roja } \\
\text { cursiva }\end{array}$ & Describir algunas acciones que realizaban los estudiantes en el software. \\
\hline $\begin{array}{l}\text { Letra negra } \\
\text { cursiva }\end{array}$ & Describir algunas observaciones o preguntas del docente investigador. \\
\hline
\end{tabular}

\section{Resultados}

\section{Análisis de la prueba inicial}

La prueba inicial fue aplicada a 70 estudiantes de $5^{\circ}$ grado de básica primaria. El análisis e interpretación de los resultados dejó como evidencia que en total de 30 estudiantes obtuvieron un desempeño bajo, y no lograron realizar de forma satisfactoria al menos uno de los ítems propuestos.

La prueba estuvo constituida por 4 ítems, que corresponden a identificación de elementos, representación y justificación. Los resultados obtenidos por la muestra de trabajo, sirvieron como punto de partida para 
las fases 1, 2 y 3 de la Etapa B de la metodología propuesta en la presente investigación. En la tabla II se muestra el análisis de los resultados por competencias evaluadas en la prueba inicial.

Tabla II. Análisis prueba inicial

\begin{tabular}{ll}
\hline Competencias & \multicolumn{1}{c}{ Resultados } \\
\hline Comunicación & $\begin{array}{l}\text { Los estudiantes no tienen una conceptualización general clara de } \\
\text { que es un poliedro regular. A través de la observación se pudo } \\
\text { establecer que los estudiantes identifican una figura } \\
\text { tridimensional pero no establecen relación a sus propiedades } \\
\text { geométricas. }\end{array}$ \\
\hline Razonamiento & $\begin{array}{l}\text { Más del } 60 \% \text { estudiantes confunden poligono con la } \\
\text { identificación de un sólido a partir de su desarrollo en el plano. } \\
\text { Tienen dificultad parar expresar cuantas caras tendría el sólido. } \\
\text { En particular se tomo como muestra de estudio el desarrollo de } \\
\text { un tetraedro y un hexaedro. }\end{array}$ \\
\hline Solución de problemas & $\begin{array}{l}\text { La mayoría de los estudiantes presentaron dificultad justificar } \\
\text { propiedades de una figura plana (cuadro, el cual constituye las } \\
\text { caras de un hexaedro). }\end{array}$ \\
\hline
\end{tabular}

De acuerdo a los resultados de la prueba inicial, se determinó el grupo de estudio, dado que contaban con el perfil que buscaba la investigación; dicho perfil está definido por estudiantes que presentaron niveles bajos en el desarrollo de la prueba. Con este grupo de estudiantes se implementó una alternativa innovadora con la incorporación de tecnologías digitales, con el software de geometría dinámica GeoGebra como entorno didáctico en el desarrollo de competencias propias de la geometría escolar.

\section{Análisis de la intervención didáctica}

Análisis preliminar. El proceso de experimentación didáctica con los estudiantes de quinto grado se llevó a cabo a través de la implementación de la siguiente secuencia de actividades con el fin de desarrollar las etapas de la geometría escolar en el estudio de propiedades de poliedros regulares que con el fin de fortalecer el desarrollo de competencias. Estas propiedades geométricas enmarcan el saber matemático en juego durante la Ingeniería Didáctica como metodología de investigación. En este sentido, en el presente artículo se muestra el análisis de una secuencia de tareas (Tabla III) en torno al estudio del Hexaedro, tetraedro, dodecaedro e icosaedro; los cuales son poliedros regulares trabajados durante la experimentación con los estudiantes.
El hexaedro es un poliedro regular comúnmente conocido como cubo y presenta la particularidad que todas sus caras son cuadradas. El cuadrado es un polígono regular que cuenta con propiedades de paralelismo entre sus lados opuestos y perpendicularidad entre sus lados consecutivos. Por otro lado, el dodecaedro es un polígono regular conformado por 12 caras pentagonales; la propiedad desarrollada con los estudiantes gira alrededor de que en poliedro regular todas sus caras son polígonos regulares, por tanto, los lados y ángulos internos de cada uno de los pentágonos que conforman el dodecaedro son congruentes. En cuanto al tetraedro e icosaedro, son poliedros formados por 4 y 20 caras respectivamente. las caras que los componen son triángulos equiláteros congruentes. 
Tabla III. Matriz de la secuencia de actividades

\begin{tabular}{llll}
\hline Actividad & Intervenciones & Objetivos \\
\hline & $\begin{array}{l}\text { Tarea } \\
\text { construcción y propiedades } \quad \text { El cuadrado, }\end{array}$ & $\begin{array}{l}\text { Reconocer que una figura bien construida conserva sus } \\
\text { propiedades luego de ampliarla o retraerla. }\end{array}$ \\
$\begin{array}{l}\text { Propiedades } \\
\text { Poliedros Regulares }\end{array}$ & $\begin{array}{l}\text { Tarea 2. El Hexaedro, un } \\
\text { poliedro regular }\end{array}$ & $\begin{array}{l}\text { Identificar las propiedades que caracterizan el hexaedro } \\
\text { en un entorno de Geometría Dinámica }\end{array}$ \\
& $\begin{array}{l}\text { Tarea 3. Desarrollo en el plano } \\
\text { de poliedros regulares }\end{array}$ & $\begin{array}{l}\text { Relacionar poliedros regulares y sus propiedades } \\
\text { geométricas con sus respectivos desarrollos en el plano. }\end{array}$
\end{tabular}

Análisis a priori. A continuación, se describe un análisis de las posibles respuestas, aptitudes y estrategias que podría haber utilizado el estudiante al momento de desarrollar cada una las tareas que se le presentaron con respecto a los poliedros regulares y sus propiedades, buscando siempre el desarrollo de competencias geométricas durante las etapas de la geometría escolar. En cada una de las tablas se muestra la intención de la tarea propuesta, la retroacción del medio, es decir, lo que el estudiante visualizó en el software luego de la acción, y la interpretación que hizo el estudiante del hecho ocurrido como se muestra en la Tabla IV.

Tabla IV. Análisis a priori

\begin{tabular}{ll}
\hline Intenciones & Tarea \\
\hline & $\begin{array}{l}\text { Acción. Lo que se le solicita al estudiante hacer en el } \\
\text { software }\end{array}$ \\
$\begin{array}{l}\text { Lo que se espera que el estudiante } \\
\text { aprenda durante el proceso }\end{array}$ & $\begin{array}{l}\text { Retroacción. Lo que el software muestra después de } \\
\text { realizar la acción }\end{array}$ \\
& $\begin{array}{l}\text { Interpretación. Lo que el estudiante deduce o concluye de } \\
\text { la tarea. }\end{array}$ \\
\hline
\end{tabular}

Análisis a priori de la Tarea 1. Mostrado en la Tabla 5, Se solicitó a los estudiantes construir un polígono regular a partir de la propiedad de perpendicularidad y paralelismo. El estudiante debería construir un cuadrado utilizando las herramientas de recta paralela y perpendicular usando el software GeoGebra como medio didáctico. La intención de la tarea fue que el estudiante potenciara su competencia de comunicación durante las etapas de la geometría de las formas y construcciones a partir de la visualización de las propiedades que componen un cuadrado, como lo son identificar que los lados de la figura son iguales, que los ángulos internos son rectos gracias a que sus lados consecutivos son perpendiculares, y que sus lados opuestos son paralelos. 
Tabla V. Análisis a priori de la tarea 1

\begin{tabular}{|c|c|c|}
\hline Intención 1 & Acción 1 & Medir los ángulos internos de la figura \\
\hline \multirow{3}{*}{$\begin{array}{l}\text { Identificar qué tipo de } \\
\text { ángulos internos tiene un } \\
\text { cuadrado }\end{array}$} & Retroacción 1 & Que cada ángulo tome un valor numérico \\
\hline & Interpretación 1 & Que todos los ángulos sean congruentes luego de realizar la acción \\
\hline & Acción 2 & Verificar que los segmentos opuestos sean paralelos \\
\hline \multicolumn{3}{|l|}{ Intención 2} \\
\hline & Retroacción 2 & Que la recta paralela se ubique sobre el segmento elegido \\
\hline $\begin{array}{l}\text { Verificar la propiedad de } \\
\text { paralelismo del cuadrado }\end{array}$ & Interpretación 3 & $\begin{array}{l}\text { Que al momento de utilizar la opción recta paralelas, la recta creada coincida } \\
\text { con el segmento opuesto al elegido inicialmente }\end{array}$ \\
\hline Intención 3 & Acción 3 & Medir las longitudes de los lados \\
\hline \multirow{2}{*}{$\begin{array}{l}\text { Establecer la congruencia } \\
\text { de los lados del cuadrado }\end{array}$} & Retroacción 3 & Que cada segmento tome un valor numérico \\
\hline & Interpretación 3 & Que los 4 segmentos del cuadrado tomen valores numéricos iguales \\
\hline
\end{tabular}

Análisis a posteriori de la Tarea 1. En el análisis a priori se solicitó al estudiante: 1) Medir los ángulos internos de la figura. 2) Verificar que los segmentos opuestos sean paralelos. 3) Medir las longitudes de los lados. A continuación, se muestran algunas de las experiencias obtenidas.

[1] E: elige la opción ángulo de la barra de herramientas y mide cada uno de los ángulos internos del cuadrado.

[2] E: observa lo sucedido luego de la acción y anota en su guía las medidas de los ángulos. figura?

[3] D: ¿Qué observas de los ángulos en la

[4] E: todos los ángulos de este cuadrado son iguales. Todos miden $90^{\circ}$.

[5] D: ¿Qué clase de ángulos son esos?

[6] E: estos ángulos son rectos

[7] D: entonces dime, si los ángulos son rectos ¿Cómo son sus segmentos?
[8] E: si los ángulos son de 90, ósea rectos, entonces los segmentos son perpendiculares, como las rectas perpendiculares.

[7] D: ¿Cómo puedes saber si los lados opuestos son paralelos o no?

[8] E: piensa por unos segundos... si son paralelos entonces puedo usar esta opción (refiriéndose a la opción recta paralela de la barra de herramientas).

[9] E: traza una recta paralela al segmento $A B$ del cuadrado que pasa por CD. También traza una recta paralela al segmento $A D$ que pasa sobre $B C$.

[10] E: mide cada uno de los segmentos que conforman los lados del cuadrado.

[11 ]D: ¿Cómo son los lados del cuadrado?

[12] E: todos los lados son iguales, tienen la misma medida. 
En el análisis a priori de esta tarea se previó que los estudiantes debían identificar qué tipo de ángulos internos tiene un cuadrado, verificar la propiedad de paralelismo del cuadrado y establecer la congruencia de los lados del cuadrado. Se observó que él estudiante realizo una buena interpretación de cada una de las retroacciones producidas de las acciones realizadas en [1], [2], [8], [9] y [10]. En este sentido con [1] y [2] el estudiante concluyó que todos los ángulos internos que conforman un cuadrado tienen la misma medida; echo que se observa en la respuesta dada en [4]. Además, con las respuestas expuestas en [6] y [7] el estudiante realizo la validación de la intensión propuesta, la cual consiste en describir que los ángulos del cuadrado son ángulos rectos, y por consiguiente sus segmentos son perpendiculares.

Por otro lado, las acciones realizadas en [8] y [9] permitieron validar que los lados opuestos de la figura son paralelos. Se observó que el estudiante no solo determinó la propiedad de perpendicularidad en un cuadrado, sino también la propiedad de paralelismo entre sus lados opuestos. Esta acción realizada por el estudiante fue rescatable, puesto que tuvo que encontrar las estrategias necesarias para solucionar la tarea. Sin embargo, otros estudiantes que no fueron capaces de usar la herramienta recta paralela para validar la intensión, se les sugirió utilizarla con el fin de que interpretaran las retroacciones generadas de la acción, y así se aproximaran a la solución de la tarea. Por último, el docente pregunta en [11] ¿Cómo son los lados del cuadrado?, a los que la gran mayoría de los estudiantes responden que los son iguales. En particular, el estudiante objeto de análisis para esta tarea, realizó una acción en [10] que interpretó y validó con la respuesta brindada en [12].

Análisis a priori de la Tarea 2. Mostrado en la Tabla 6; esta tarea tuvo como objeto de estudio el desarrollo de la competencia de razonamiento durante la etapa de la Geometría de las justificaciones. El software GeoGebra es el medio didáctico en el proceso de enseñanza, que trabaja el saber específico de poliedros regulares y sus propiedades. El estudiante debió construir un hexaedro utilizando la vista en 3D o de tercera dimensión del programa, para ello retoma la construcción del cuadrado desarrollado a partir de sus propiedades, debido a que esta figura conforma cada una de las caras del hexaedro.

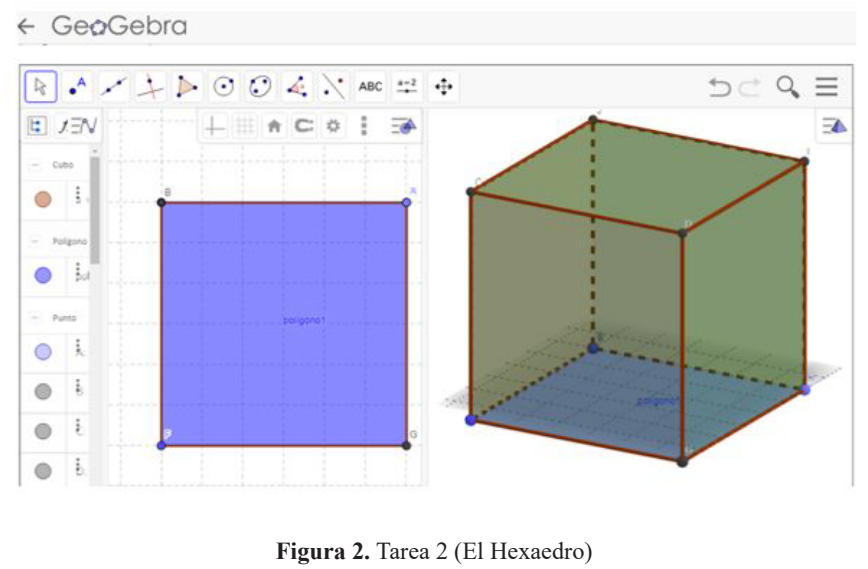

Con la construcción del hexaedro el estudiante debió argumentar porque la figura es un poliedro regular, y que propiedades lo componen 


\begin{tabular}{|c|c|c|}
\hline \multirow{9}{*}{$\begin{array}{l}\text { Intención } \\
\text { Identificar propiedades del } \\
\text { hexaedro }\end{array}$} & Acción 1 & Girar el hexaedro en todos los ángulos posibles \\
\hline & Retroacción 1 & Se puede ver el hexaedro desde diferentes ángulos \\
\hline & Interpretación 1 & $\begin{array}{l}\text { Que cada ángulo en que se observa el poliedro permita visualizar las } \\
\text { caras que lo conforman }\end{array}$ \\
\hline & Acción 2 & $\begin{array}{l}\text { Medir las longitudes de las aristas del hexaedro, y los ángulos } \\
\text { comprendidos entre cada par de aristas }\end{array}$ \\
\hline & Retroacción 2 & Que las longitudes y los ángulos tomen un valor numérico \\
\hline & Interpretación 2 & Que los valores numéricos de las longitudes y ángulos sean congruentes \\
\hline & Acción 3 & Calcular el área de las caras del poliedro \\
\hline & Retroacción 3 & En cada figura el área es un valor numérico \\
\hline & Interpretación 3 & Que los valores numéricos de las áreas sean congruentes \\
\hline
\end{tabular}

Análisis a posteriori de la Tarea 2: En el análisis a priori se estableció la tarea de girar el hexaedro en todos los ángulos posibles, medir las longitudes de las aristas del hexaedro y los ángulos comprendidos entre cada par de aristas; además, calcular el área de las caras del poliedro.

Las siguientes son algunas concepciones de como son las caras del poliedro y algunas características de un hexaedro por parte de los estudiantes:

[1] G: mide algunas de las aristas del hexaedro. Observa mientras arrastra la figura. Al final responde

[2] G: todas las caras del hexaedro son cuadrados, ya habíamos visto que sus ángulos son rectos y sus lados son iguales, Por eso el polígono es regular siempre

[3] G: calcula el área de tres caras del hexaedro y arrastra la figura

[4] G: los áreas son iguales también, claro por son cuadrados iguales.
Además, en cuanto a las propiedades del solido construido se observó una congruencia en la respuesta grupal:

[5] G: todas las caras del poliedro son iguales, por eso sus ángulos y aristas también son iguales. Por eso es que las caras de esta figura 3D son polígonos regulares y son cuadrados.

En el análisis a priori de esta tarea se preveía que los estudiantes al momento de mover los vértices del hexaedro debían observar que sus caras eran congruentes, puesto que son cuadrados. Se observa por las acciones en [1] y [3] y las interpretaciones en [2], [4] y [5] que la actividad es una auténtica situación a-didáctica, puesto que el grupo logró desarrollar la actividad sin ayuda del docente más allá de solo hacer intervenciones generales en el aula virtual para explicar pasos de las construcciones.

Análisis a priori de la Tarea 3. Mostrado en la Tabla 7. La tarea tuvo como objetivo relacionar poliedros regulares y sus propiedades geométricas con sus respectivos desarrollos en el plano. La tarea 3 constituye la última de la secuencia que busca desarrollar la etapa de las justificaciones en la geometría escolar. 


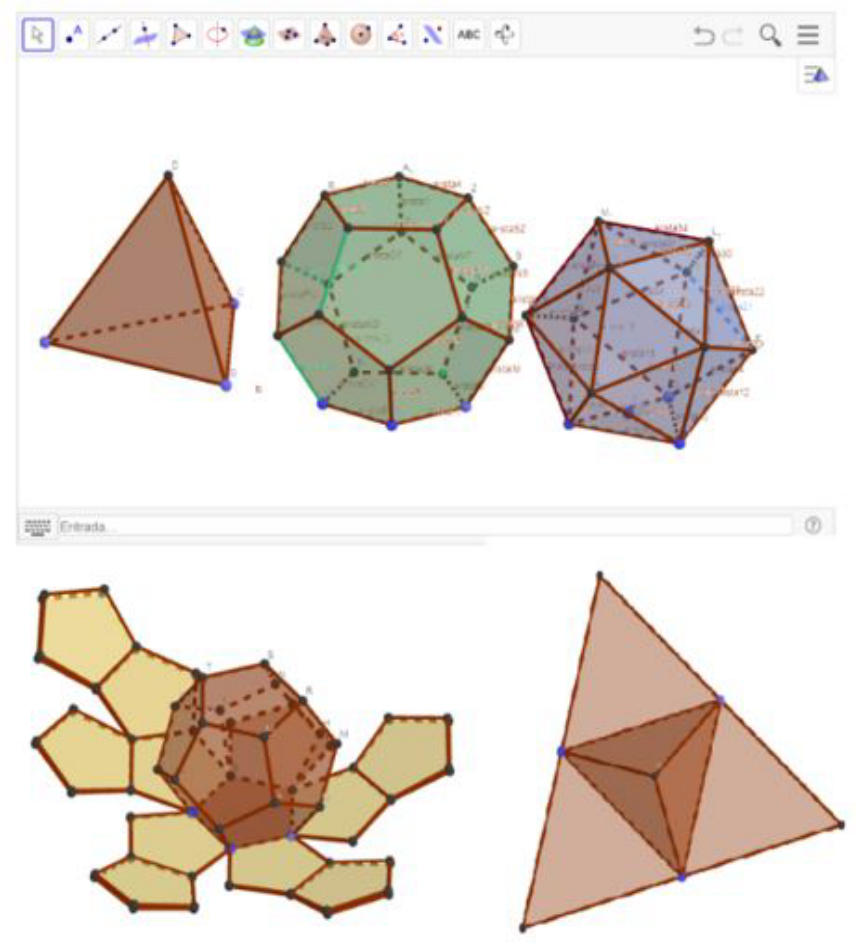

Figura 3. Tarea 3 (Desarrollos en el Plano)

En esta tarea los estudiantes debían argumentar porque el tetraedro, dodecaedro e icosaedro son poliedros regulares a partir de propiedades que identifiquen en la figura luego de realizar las construcciones solicitadas.

Tabla VII. Análisis a priori de la tarea 3

\begin{tabular}{lll}
\hline & Acción 1 & Realizar el desarrollo en la vista 3D de los poliedros \\
Intención & Retroacción 1 & Que se muestre el desarrollo de los poliedros \\
$\begin{array}{l}\text { Establecer relaciones entre los } \\
\text { elementos que conforman las } \\
\text { caras de los poliedros regulares }\end{array}$ & Interpretación 1 & $\begin{array}{l}\text { Que los polígonos visualizados en el desarrollo sean congruentes con las } \\
\text { caras del poliedro }\end{array}$ \\
& Acción 2 & $\begin{array}{l}\text { Medir los ángulos y lados de los polígonos visualizados en los } \\
\text { desarrollos. }\end{array}$ \\
& Retroacción 2 & Que las medidas se reflejen como valores numéricos \\
& Interpretación 2 & Que todas las medidas sean congruentes \\
\hline
\end{tabular}

Análisis a posteriori de la Tarea 3. Los estudiantes debieron hacer el desarrollo de un tetraedro, dodecaedro e icosaedro a partir de los polígonos regulares que lo conforman. La tarea consistió en realizar el desarrollo en la vista 3D de los poliedros, medir los ángulos y lados de los polígonos visualizados en los desarrollos. 
A continuación, se muestra la respuesta de un grupo de estudiantes en cuanto a las caras y en particular, a las propiedades de los poliedros:

[1] G: respuesta del grupo luego de realizar las acciones propuestas en la tarea

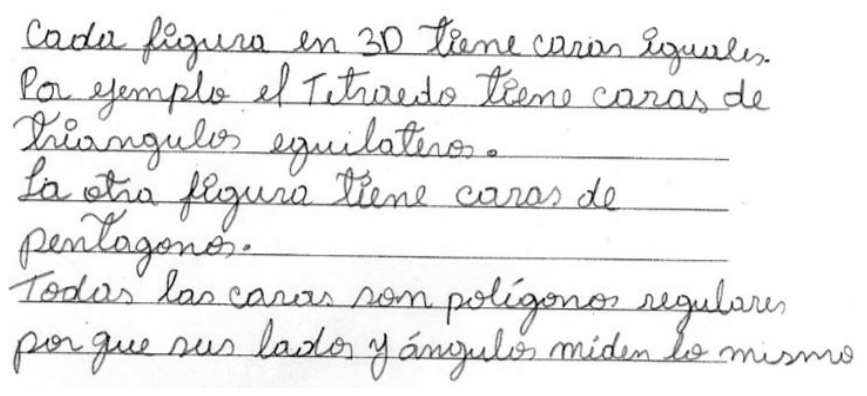

Figura 4. Respuesta de un grupo analizado

[2]D:¿entonces que es un poliedro regular?

[3] G: respuesta del grupo
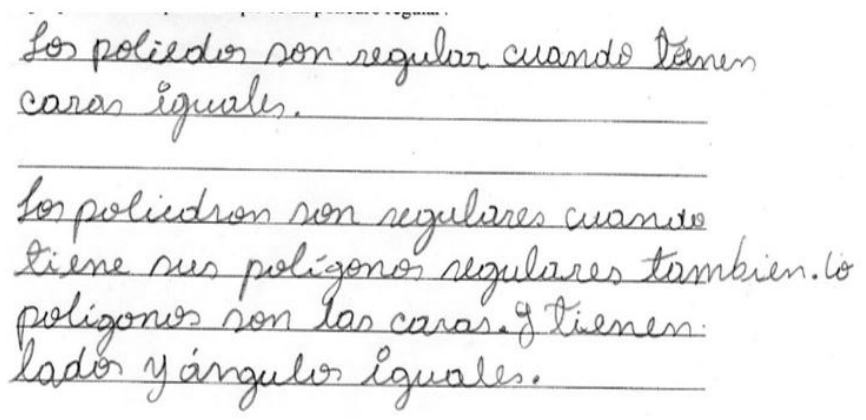

Figura 5. Respuesta del grupo analizado

En el análisis a priori de esta tarea se previó que los grupos debían argumentar o dar una conceptualización de que es un poliedro regular a partir de las propiedades que lo conforman. Se observó por las acciones en [1] y [3] que el grupo justifica que las caras de los poliedros son iguales, en el caso del tetraedro son triángulos equiláteros. En general argumentaron que en todos los desarrollos sus caras son polígonos regulares. Por consiguiente, afirmaron que un poliedro es regular cuando sus caras son iguales y además deben ser polígonos regulares.

\section{Conclusiones}

La presente investigación responde a la hipótesis planteada acerca de la eficacia del uso de las tecnologías digitales en procesos de enseñanza y aprendizaje que permitan desarrollar competencias lógico matemáticas en los estudiantes, en especial en el desarrollo de competencias propias de la geometría enmarcadas dentro del estudio de las etapas de la geometría escolar. En especial, la secuencia actividades a partir situaciones a-didácticas desarrolladas con los estudiantes de quinto grado de las Instituciones Educativas objeto de estudio, permitió potenciar las competencias de comunicación, razonamiento y solución de problemas necesarias para el desarrollo del pensamiento lógico matemático.

Estas actividades que se trazaron bajo la perspectiva teórica de una situación a-didáctica, la cual es la interacción entre un sujeto y un medio para resolver un problema. En tal medida el maestro debe ser preparar la situación a-didáctica seleccionando de forma cuidadosa el medio y el problema que planteará a los alumnos. El logró de la presente investigación estuvo en poder diseñar situaciones a-didácticas que garantizaron el uso de las tecnologías digitales (software GeoGebra) no sólo como una herramienta computacional, sino como un ambiente completo de aprendizaje; permitiendo visualizar, construir y justificar propiedades geométricas por parte de los estudiantes.

En general, se mostró el diseño, implementación y análisis de actividades didácticas para alcanzar el objetivo de la investigación, desarrollar competencias durante las etapas de la geometría escolar en situaciones reales de clase, incorporando las tecnologías digitales en la enseñanza de la geometría de una forma asertiva y dinámica. Además, el análisis de las actividades propuestas fue posible y fundamentado gracias al uso de la Ingeniería Didáctica como referente metodológico de investigación y que es usada principalmente en 
investigaciones de incorporan tecnologías digitales y diseño de estrategias innovadoras en su desarrollo.

El desarrollo de competencias durante las etapas de la geometría escolar permite a nivel específico potenciar el desarrollo del pensamiento espacial en los estudiantes, puesto que son capaces de visualizar propiedades de figuras planas y tridimensionales, en particular de un poliedro regular; argumentar fenómenos geométricos a partir de sus propiedades. Lo anterior concuerda con lo establecido en el documento de los Lineamientos Curriculares en Colombia, donde se define el pensamiento espacial como "... el conjunto de los procesos cognitivos mediante los cuales se construyen y se manipulan las representaciones mentales de los objetos del espacio, las relaciones entre ellos, sus transformaciones, $\mathrm{y}$ sus diversas traducciones o representaciones materiales" (MEN, 1998, p 56).

Encuantoal desarrollo de la etapa de la geometría de las formas, construcciones y justificaciones, estas se dan cuando el docente es capaz de desarrollar en sus estudiantes estrategias que les permitan en primera medida visualizar formas, objetos y propiedades geométricas. Lo anterior se evidenció con los análisis a priori y posteriori de las tareas en el proceso de intervención didáctica.

Cada una de las actividades desarrolladas durante la presente investigación estuvieron mediadas por el software de Geometría Dinámica GeoGebra. En efecto, la Geometría Dinámica tiene un potencial didáctico que debe ser explotado por los docentes de matemáticas, con el fin de crear nuevas estrategias de enseñanza y aprendizaje que enriquezcan el quehacer pedagógico. Se puede afirmar, que el uso de tecnologías digitales está ligado a los avances que está presentando la sociedad a nivel educativo y que deben responder a la motivación y satisfacción que sienten los estudiantes al trabajar con tecnología (Rodríguez, López y Mortera, 2017) y a la adaptación de los contenidos a los nuevos estudiantes o nativos digitales (Chávez 2015).
Se motiva a los docentes de matemáticas diseñar situaciones a-didácticas para el desarrollo de sus clases y en especial, a apropiarse e implementar actividades mediadas por software de Geometría Dinámica, que potencien el pensamiento espacial, despierten interés de los estudiantes y trasciendan las barretas de tablero, cuaderno y lápiz con que se ha enseñado las matemáticas de forma tradicional.

\section{Referencias}

Acosta, M. (2010). Enseñando transformaciones Geométricas con software de Geometría Dinámica. Documento presentado en el $11^{\circ}$ Encuentro Colombiano Matemática Educativa. Colombia: Bogotá

Acosta, M., Monroy, L., \& Rueda, K (2010). Situaciones a-didácticas para la enseñanza de la simetría axial utilizando Cabri como medio. Revista Integración. Universidad Industrial de Santander. Vol. 28, No. 2, pp. 173-189

Acosta, M (2017). Enseñando geometría con tecnología digital: una propuesta desde la teoría de las situaciones didácticas. Universidad Distrital Francisco José de Caldas. Colombia. Bogotá

Artigue, M. (1995). Ingeniería didáctica en educación matemática: Un esquema para la investigación y la innovación en la enseñanza y el aprendizaje de las matemáticas. Colombia. Bogotá

Badia, A., Chumpitaz. L.,Ibarra, A. \& Llata, D. (2010). Niños nativos digitales en la sociedad del conocimiento: acercamientos conceptuales a sus competencias. Razón y Palabra. No. 72, pp 1-24. Disponible en http://www.razonypalabra. org.mx/N/N72/Varia_72/14_Ibarra_72.pdf

Camargo, L. \& Acosta, M. (2012). La geometría, su enseñanza y su aprendizaje. Tecné, Episteme y Didaxis. (32). pp. 1-10. Disponible en: http:// 
revistas.pedagogica.edu.co/index.php/TED/ issue/view/197/showToc

Clemens, D. \& Battista, M. (1992). Geometry and Spatial Reasoning. En D. Grouws (Ed.). Handbook of Research on Mathematics teaching and Learning: A Project of the National Council of Teachers of Mathematics (pp. 34-67). New York: NCTM

Conde-Carmona, R. J., \& Fontalvo-Meléndez, A. A. (2019). Didáctica del teorema de Pitágoras mediada por las TIC: el caso de una clase de Matemáticas. Trilogía Ciencia Tecnología Sociedad, 11(21), 255-281. https://doi. org/10.22430/21457778.1187

Conde-Carmona, R. J., Samper-Taboada, A. P., \& Camacho-Mendoza, L. J. (2020). Experimental mathematics practices and mathematical modeling in math teachers in training for the construction of proposals for new ways of teaching quadratic function. Espacios, 41(30), 282-291

Hernández, R., Fernández, C. \& Baptista, P. (2014). Metodología de la investigación (6aed.). México, D.F., México: McGraw-Hill Interamericana

Janssen N, Lazonder AW (2015). Implementing Innovative Technologies Through Lesson Plans: What Kind of Support Do Teachers Prefer?. Journal of Science Education and Technology 24:910-920 DOI: 10.1007/s10956015-9573-5

Lucumi, P. \& González M. (2015). El ambiente digital en la comunicación, la actitud y las estrategias pedagógicas utilizadas por docentes. Tecné, Episteme y Didaxis. (37). pp. 109 - 129

Ministerio de Educación Nacional. MEN (2015). Matrices de Referencia. Colombia. Bogotá
Mulder YG, Lazonder AW, De Jong T, Anjewierden A, Bollen L (2012). Validating and optimizing the effects of model progression in simulation-based inquiry learning. Journal of Science Education and Technology 21:722-729. DOI:10.1007/ s10956-011-9360-x

Padilla Escorcia, I. A., \& Junior Conde-Carmona, R. (2020). Uso y formación en TIC en profesores de matemáticas: un análisis cualitativo. Revista Virtual Universidad Católica del Norte, (60)

Rodríguez, R., López, B. \& Mortera, F. (2017). El video como Recurso Educativo Abierto y la enseñanza de Matemáticas. Revista Electrónica de Investigación Educativa, 19(3), 92-100. https://doi.org/10.24320/redie.2017.19.3.936

Rueda, Niño \& Acosta. (2013). Automatización de decisiones didácticascon el software Cabri elem. Pp. 572-574. Universidad Industrial de Santander. Colombia

Squire KD, Jan M (2007). Mad City Mystery: Developing Scientific Argumentation Skills

with a Place-based Augmented Reality Game on Handheld Computers. Journal of Science Education and Technology 16(1): 5-29. DOI: 10.1007/s10956-006-9037-z

Van Dijk AM, Lazonder AW (2013) Scaffolding students' use of learner-generated content in a technology-enhanced inquiry learning environment. Interact Learn Environ. doi:10.10 80/10494820.2013.834828

Vargas, V., Escalante, C. \& Carmona, G. (2018). Competencias Matemáticas a través de la implementación de actividades provocadoras de modelos. Educación Matemática. 30(1), 213236. DOI: $10.24844 / \mathrm{EM} 3001.08$ 